\title{
Web Load Balancing Method Based On Resource Request Division
}

\section{Author:Zhanfang Chen ${ }^{1}$,Xiaoming Jiang ${ }^{2}$ Yingchao Zhang ${ }^{3}$, Mubarak Albarka Umar ${ }^{4}$}

\author{
Affiliation: Changchun University of Science and Technology $y^{1,2,3,4} 130022$ China \\ E-mail: jeffy2010@126.com ${ }^{1}$; \\ jiangxiaoming@cust.edu.cn ${ }^{2} ; 2019100620 @$ mails.cust.edu.cn ${ }^{3} ; 12018300037 @$ mails.cust.edu.cn ${ }^{4}$
}

<DOI:10.26821/IJSHRE.8.9.2020.8910 >

\begin{abstract}
The main function of WEB load balancing is to receive requests sent from clients, and then dynamically allocate requests to the available nodes in the backend. The resources of the backend node server are called according to the rules and policies of the load balancer. Comparing functions, the static load balancing algorithm is an important part of the load balancing algorithm. The characteristics are that the system consumes less resources and is easier to implement. The principle of the static load balancing algorithm is easy to understand, simple to implement, and distributes requests in random and polling. The randomness cannot be predicted, resulting in poor load balancing effect, and the polling regularity is too strong to achieve the corresponding load balancing effect. In response to the above problems, this chapter mainly classifies the received HTTP requests to optimize the load balancing algorithm . A WEB load balancing algorithm based on resource request division is proposed. The request resources are divided into static request resources and dynamic request resources, and the static request resources are cached in memory to improve access efficiency.
\end{abstract}

Keywords: WEB load balancing algorithm; Static load balancing algorithm.

\section{INTRODUCTION}

The main function of WEB load balancing is to receive the request sent from the client, and then dynamically allocate the request to the available node at the back end. According to the rules and policies of the load balancer, the resource of the back end node server is invoked. The load balancing tochnology plays an incomparable role in the server cluster. Load balancing technology can improve the efficiency of back-end node server and application throughput, and the application of load balancing technology is an efficient and convenient way for users.

Static load balancing algorithm is an important part of load balancing algorithm. It allocates resources according to the task allocation strategy in the computer rather than consider factors such as the load condition of the back-end nodes. the advantage of the static load balancing algorithm is that the system consumes less resources and it easier to implement. In addition, an algorithm is involved dynamic load balancing algorithm, which acquires node information periodically and forms a dynamic return task allocation strategy based on the task allocation strategy.

Static load balancing algorithm principle is easy to understand, to implement is easy, according to random and polling to distribute requests. The randomness cannot be predicted, which leads to poor load balancing effect, and the polling regularity is too strong to achieve the corresponding load balancing effect.Dynamic load balancing is realized by using node feedback parameters, request response time parameters and real-time connection number parameters as the basis.It is mainly suitable for heterogeneous clusters, high hit ratio cache data clusters and response time load clusters.Static load balancing algorithm and dynamic load balancing algorithm treat all requests uniformly without considering 
Volume 8 Issue 9 September 2020

the classification of requests. HTTP requests can be divided into dynamic resource requests and static resource requests .In view of the above problems, this chapter mainly classifies and processes the received HTTP requests so as to optimize the load balancing algorithm.

\subsection{REQUEST RESOURCALLOCATION}

This chapter divides the request resources into dynamic request resources and static request resources.As shown in figure 4.1, there are no other program files that can be executed for the static request resource. Once the static resource is made, the content will not change. The display of the static resource to the user is always the same, and the result of request from different browsers is always the same.Static resources do not connect to the database, may not be able to do and user data information interaction, if you want to modify the content, you must modify the source code, and then upload to the server.Static request resources include audio, images, and other data files that do not change their content, as well as static web pages.

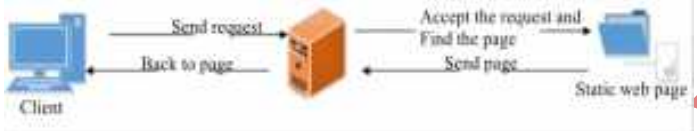

Figure 1.1 Static Resource Request Process As shown in figure 1.2, dynamic request resources contain not only common HTML tags, but also executable program files, which can dynamically generate returned pages based on different inputs and requests.Such as access. Net, PHP, JSP and other program files are dynamic resource requests.

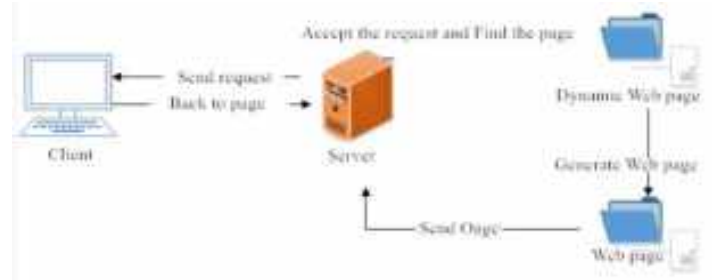

Figure1.2 Dynamic Resource Request Process

There is a difference between the server resources consumed by static and dynamic resources when the server responds to these HTTP requests.Therefore, targeted processing of various resource requests not only reduces the consumption of server resources but also improves the response speed.In addition, the load balancer specifically divides HTTP request resources into dynamic and static resources.Not only are the two request resources different in terms of the requested resource, but also the server has a great difference in processing the request and response.Dynamic resource is the response data file formed by the program after processing, and then the data file is combined into the final data file to be sent to the requesting user. In addition, the file data is readable according to the difference of request parameters, so it is not easy to cache.And at the same time, the server in the treatment of the static resources, first check whether there is the resource cache area, if there is no resources else from the server disk access, and according to the further Recently informs the scheduling algorithm to written in the cache, if any, resource cache areacan obtain directly from the cache and return of this static resource, in this way can improve the efficiency of request and response, making full use of server nodes working ability.

\subsection{CAlCulation OF PROCESSING CAPACITY}

Based on the dynamic load balancing strategy, this chapter proposes an application load balancing method based on resource request partitioning.After consulting relevant materials, we found that at this stage, four indicators were used to measure the load status of server nodes: CPU share, memory capacity, disk I/O rate and network throughput.In this chapter, the remaining CPU usage, memory and network capacity, and disk I/O usage are used as the parameters to evaluate the load status of the load balancing strategy.

After a lot of references, we found that the linear weighted function evaluation model has obvious advantages in load model research, which not only saves server resources but also is easy to implement.Given these advantages, a linear weighting method is preferred in this chapter to represent the load condition of the server nodes. The specific form is as follows:

$$
f\left(X_{1}, X_{2}, \ldots, X_{n}\right)=k_{1} \times X_{1}+k_{2} \times X_{2} \cdots k_{n} \times X_{n}
$$

Generally, formula (1.3) is used to calculate and quantify the load condition of nodes.Is load information, which reflects the priority of load information.The vector composed of all coefficients is involved index vector. Only by determining this vector can the evaluation function be determined and the priority of load information be reflected.In this chapter, the remaining CPU usage, memory usage, network usage, and disk I/O usage are used as parameters to evaluate the load status of the load balancing strategy.The evaluation function is as follows: 
Volume 8 Issue 9 September 2020

$$
L=k_{c} \times L_{c}+k_{m} \times L_{m}+k_{d} \times L_{d}+k_{n} \times L_{n}(\mathbf{1 . 4})
$$

Refers to server load, refers to CPU utilization, refers to the remaining capacity after memory use, refers to the remaining amount of disk $\mathrm{I} / \mathrm{O}$, refers to the remaining throughput of the network, respectively represents the index coefficient of CPU, memory, disk I/O and four corresponding resources of the network.

\section{MIXED RESOURCE REQUEST LOAD}

\subsection{Static resource load}

Static resource requests generally do not change the contents of the requested files, which are mostly stored on the server disk. The server consumes CPU, disk I/O, memory capacity, and network throughput while processing static resource requests.

1) After receiving the static resource request, the server application will obtain the corresponding static resource of the server and process the corresponding static resource through the access address. All these processes will consume the server resource. At this time, the CPU core number and CPU main frequency are inversely proportional to the load generated by the CPU, Reference Formula:

$$
L_{c} \propto \frac{1}{\alpha f}(\mathbf{2 . 1})
$$

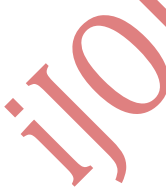

2) Reading static resource files will consume the disk $\mathrm{I} / \mathrm{O}$ rate. If the static resource files are too large and exceed the access range of track addressing space, additional access to other tracks on the disk will be required, which will consume the disk addressing time.So the static file size and disk I/O rate.Reference Formula:

$$
L_{d} \propto \frac{f s}{D}
$$

3) Static resource files are generally stored in the cache and can be read directly from the cache when the client accesses the static resource.If the static resource is accessed for the first time or is not in the cache, the corresponding file on disk needs to be read.The static resource file is then stored in an in-memory cache.Since the memory capacity is limited, LRU algorithm is used to replace the cache information.Static resource files that are larger than a certain size are not cached in memory. Such files need to be read to disk every time they are accessed, and the size of the memory capacity for the server is proportional to the size of the cached file.Therefore, the file size and memory capacity are shown in the formula:

$$
L_{m} \propto \frac{f s}{M}
$$

4) Static resource file transmission consumes network bandwidth. The larger the file, the larger the network throughput required. On the contrary, the smaller the network throughput, the larger the file load, and the larger the network bandwidth load. Therefore, the file size and network throughput, reference formula.

$$
L_{n} \propto \frac{f s}{N}
$$

In summary, the calculation formula (2.4) of the static resource load model at time $t$ is the number of static resouree requests at time $t$, which is the adjustment index coefficient of the resource load at time $t$. If it is 0 , the Cached static file is read, and no load is generated on the disk at this time. If it is 1 , the memory does not cache static files and needs to read the disk. At this time, the disk generates a load.

$$
L_{s}(t)=f(t)\left[k_{1} \cdot L_{c}+\lambda k_{2} \cdot L_{d}+k_{3} \cdot L_{m}+k_{4} \cdot L_{n}\right]=f(t)\left[\frac{k_{1}}{\alpha f}+\frac{\lambda k_{2} f_{s}}{D}+\frac{k_{3} f_{s}}{M}+\frac{k_{4} f_{s}}{N}\right]
$$

\subsection{Dynamic resource load}

Dynamic resource load is closely related to CPU utilization, memory capacity, disk I/O rate, and network throughput, but compared to the static resource load model, parameters directly determine the query size and file size, because HTTP requests and processing differences lead to different dynamic resource generation.For example, if a specific parameter is selected to access the database, specific data will be obtained. As the parameter changes, the amount and type of data obtained will also change.In the same way, dynamic resource requests change as parameters change.Therefore, compared with static resource requests, processing dynamic resource requests consumes more resources. Dynamic resources are usually not written into the cache and have certain uncertainties. Therefore, dynamic resource requests are relatively complex and cannot be quantitative.

This chapter divides the resources requested over HTTP into dynamic resource requests and static resource requests. The dynamic resource load is obtained by subtracting the total load of the server node from the static 
Volume 8 Issue 9 September 2020

resource load.This method can accurately reflect the dynamic resource load.Previous load model metrics were based on server response time and server connections. These parameters do not take into account the actual hardware operation of the server node in all aspects, and their accuracy is poor.

The total load of the four index parameters using CPU utilization, memory capacity utilization, disk I/O rate and network throughput utilization at time $\mathrm{t}$ is shown in formula (2.6), where is the weight of the index parameters:

$$
L(t)=\left[x_{1} \cdot v(C(t))+x_{2} \cdot v(M(t))+x_{3} \cdot v(D(t))+x_{4} \cdot v(N(t))\right]
$$

Through the total load and the static resource load, the dynamic load of the node at time $t$ can be calculated, reference the formula:

$$
L_{d}(t)=L(t)-L_{s}(t)
$$

\subsection{Mixed Resource Request Distribution Processing}

Through the dynamic resource load model and the static resource load model, the server calculates the total load of the node, and then the load scheduler processes the resource request according to the load. It should also be noted that the load scheduler is enabled to receive the status information of the server node once in a time period and the general algorithm is enabled to control one server to process these resource requests. When the traffic is concentrated, it may cause the server node Overload, which can lead to server node downtime.

The content of this chapter is to establish a top-down mixed resource request distribution process to solve the above problems: the distribution process initializes a comparison table containing the correspondence between keys and servers when distributing static resource requests, and improves the response speed of static resource requests; When distributing dynamic resource requests, establishing a kind of sampling period to distribute to multiple node servers to effectively prevent the problem of overloading the nodes of centralized access servers.

\subsection{Static resource request distribution processing}

In order to improve the speed of users accessing static resources, build a key with the first letter of the static resource file name (such as a, b, c ... z), assuming all static file names are composed of letters, and the values correspond to the back-end nodes, the specific structure is shown in the table 2.8:

\begin{tabular}{cc}
\hline Key(File initials) & Value (Backend server node) \\
\hline $\mathrm{a}$ & Server $i_{\mathrm{a}}$ \\
$\ldots$ & $\ldots$ \\
$\mathrm{Z}$ & Server $_{\mathrm{z}}$ \\
\hline
\end{tabular}

\section{Table 2.8 comparison table of load balancers}

After the server receives the request, the load balancer obtains the first letter of the received static resource file name, and maps the requested resource to the back-end server node one-to-one through a comparison table. If the requested resource is not in the comparison table, the first letter of its request file is allocated to the server node with the smallest current load, and the key value in the load balancer comparison table is changed to update the current server allocation status.

$$
[a, b, c, \ldots, z]=\left\{\frac{i_{a, b, c, \ldots z} ; \eta_{i}(t) \leq \beta}{\min (\eta) ; \eta_{i}(t)>\beta}\right.
$$

When accessing a static resource request for the first time, the load balancer randomly selects a background server node. Since this reduces the probability of cache hits, restart the lookup table. First, determine the static file names in alphabetical order. In order to avoid the problem of overloading the server caused by excessive traffic and ensuring that each server can get requests for static resources, a "dragon tail" method is proposed to allocate static resource requests. . For example, the first letter a of the file name is assigned to server 1 , and the first letter $b$ of the file name is assigned to server 2. Until each server can be assigned a static resource request, if there are still static resource requests, the static is assigned from the last server. Resource requests, until the first server, until the static resource requests with a file name beginning with $\mathrm{z}$ are assigned to a specific server, reference Table2.10: 
Volume 8 Issue 9 September 2020

\begin{tabular}{cc|}
\hline Key(File initials) & Value (Backend server node) \\
\hline a & Serverl \\
$\ldots$ & $\ldots$ \\
$g$ & Server n \\
h & Server n \\
$\ldots$ & $\ldots$ \\
z & Serverl \\
\hline
\end{tabular}

Table 2.10Load balancer comparison table

\subsection{Dynamic resource request distribution processing}

In the previous section, we introduced the load balancer comparison table for assigning static resource requests to server nodes. However, due to the diversity of request resource files for dynamic resource requests, the resources occupied by these request resource files are also unknown, so It cannot be all assigned to one server node. Not only can it not achieve the effect of load balancing, it may also cause a single point of failure. As shown in Figure 2.11, it is assumed that the load balancer sampling period is $\mathrm{T}$, which changes exactly in a time period. At the moment, the smallest dynamic resource load in the back-end server node is server c. After that, it is assumed that dynamic resources are in the time period. Requests are sent to the server in a centralized manner. At this time, since the dynamic resource load of server $\mathrm{c}$ is the smallest, all requests are distributed to server $\mathrm{c}$ for processing by the load balancer, causing server $\mathrm{c}$ to be overloaded.
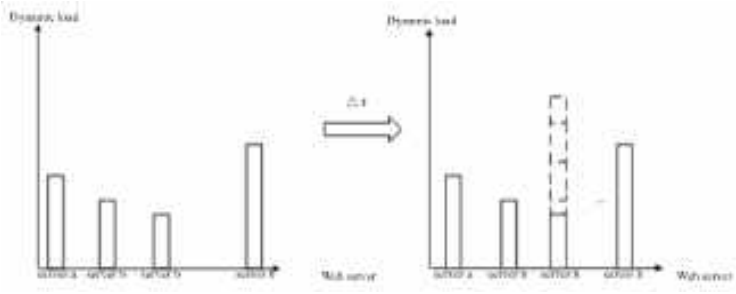

Figure 2.11Time load variation

In view of this situation, the load balancer allocates the overall (dynamic resource request) to each server node within $\mathrm{T}$. Through the introduction of the dynamic resource load, the average value of the load balance is determined according to the mathematical expectations, reference formula (2.12). Judging the effect of load balancing based on variance. According to the negative deviation degree as the distribution basis, reference formula (2.13), parametern is the number of server nodes and the dynamic load of the server node:

$$
E(t)=\frac{\sum_{k=1}^{n} L_{d_{k}}(t)}{n}=\frac{\sum_{k=1}^{n}\left(L_{k}(t)-L_{s_{k}}(t)\right)}{n}
$$

$$
\mathrm{D}(t)=L_{d_{\mathrm{i}}}(t)-E(t)
$$

$$
E_{d}(t)=\frac{\sum_{i=1}^{n}\left|L_{d_{i}}(t)-E(t)\right|}{n}
$$

From the formulas (2.13) and (2.14), it can be known that at parameter time $t$, the larger the negative absolute value, the smaller the dynamic load and the smaller the smaller, the better the load balancing effect of the entire cluster.

The above algorithm can well solve the problem of distributing dynamic resource requests to the same server node, reducing the overload of back-end server nodes during the entire access request stage, and the values are getting smaller and smaller, and the load rate of each server is getting closer to the average Load, so that the load balancer can achieve the ideal load balancing effect.

\section{EXPERIMENTAL SIMULATION AND ANALYSIS}

\subsection{Experimental preparation}

This chapter uses experiments to verify the performance of the algorithm proposed above. In order to improve the comprehensiveness and reliability of the experiment, a static load balancing algorithm-weighted polling algorithm (WRR) and a dynamic load balancing algorithm-minimum connection are selected for experimental simulation Number algorithm (LC), comparing the WRR algorithm and the LC algorithm with the algorithms in this chapter through the load balancer average load and CPU utilization two parameters.

In order to simplify the experimental environment, this chapter uses a network topology environment similar to Chapter 3, as shown in Figure 3.1. 
Volume 8 Issue 9 September 2020

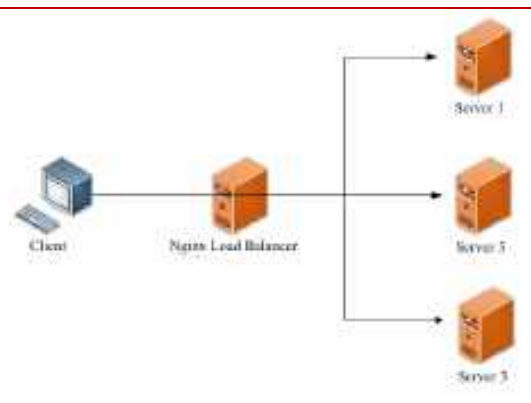

Figure 3.1 Web cluster architecture diagram

In this chapter, Nginx is adopted as the reverse proxy and load balancing server ofWEB cluster. As a load balancer, Nginx is very efficient and can withstand massive concurrent requests.Both the load balancer and the back server run on Linux, and Linux is managed and configured on a file-based basis, so when you get performance information about each node, you just need to read it directly from the file through the cat command.

The load balancer is used to calculate the total request load, static request load, and dynamic request load of the server node. Based on the resource request division, the dynamic resource request is calculated by calculating the expected dynamic request load and dynamically allocates resources. The static resource request is improved through the comparison table Cache hit rate. As shown in Figure 3.2.

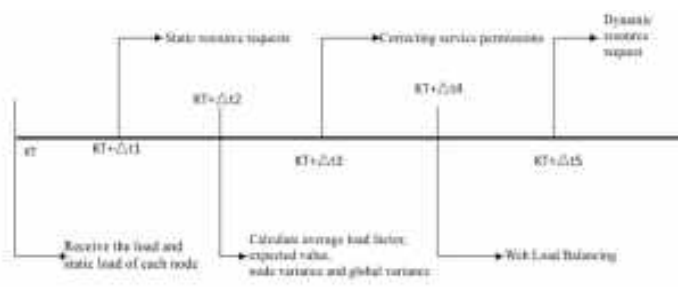

Figure 3.2 Working timing diagram of load balancer

\subsection{Experimental conclusion and analysis}

The performance of WEB cluster load balancing is mainly reflected in two factors: the average throughput of the WEB cluster load and the CPU utilization. The average throughput represents the processing capacity of the WEB cluster, and the CPU utilization represents the resource utilization of the load balancer. The performance of the cluster in the WEB load balancing algorithm based on resource request partitioning, combined with the comparison of the WRR algorithm and the LC algorithm, that convenient to more intuitively reflect the advantages of the algorithm in this chapter.
The average throughput is shown in Figure 3.3. At the beginning, the server throughput was 0. As user requests increased, the throughput also increased. Subsequently, the increase in user requests and the WEB cluster throughput reached a balance. The average throughput of the WEB cluster has stabilized. It can be seen from the figure that at the beginning of the three algorithms, the steady increase with the increase in requests. As time changes, the algorithm in this chapter calculates and divides the requested resources through the load balancer, and caches the static resources into memory. It requests the load balancer to distribute dynamic resources. Through mixed resource request distribution processing, each node caches certain static resources, gradually reduces static requests, and finally brings the WEB cluster to a load balanced state. At this time, the advantages of the algorithm in this chapter are obvious. The average throughput of the algorithm is higher than the average throughput of the WRR algorithm and the LC algorithm.

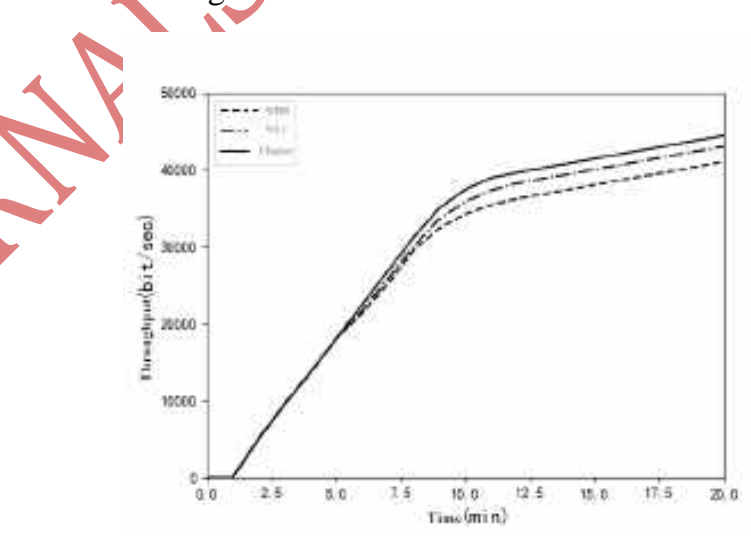

Figure 3.3 average throughput

The CPU utilization is shown in Figure 3.4. It can be seen from the figure that the weighted polling algorithm (WRR) has the highest CPU utilization, followed by the least connection algorithm (LC), and the algorithm has the lowest CPU utilization. There is no difference in CPU utilization among the first three algorithms. Compared with the three algorithms, but compared to the three algorithms, WRR is based on a static load balancing algorithm. The resource occupation is also very small, but with the increase in user requests, the WEB cluster becomes unbalanced, reducing work efficiency, affecting the workload of the load balancer, and increasing CPU utilization. This situation seriously affects the load balancing of the WEB cluster To improve the above problems, it is necessary to select the appropriate load balancing algorithm based on the complexity of the Web cluster load algorithm and the overall load balancing effect of the Web cluster. 
Volume 8 Issue 9 September 2020

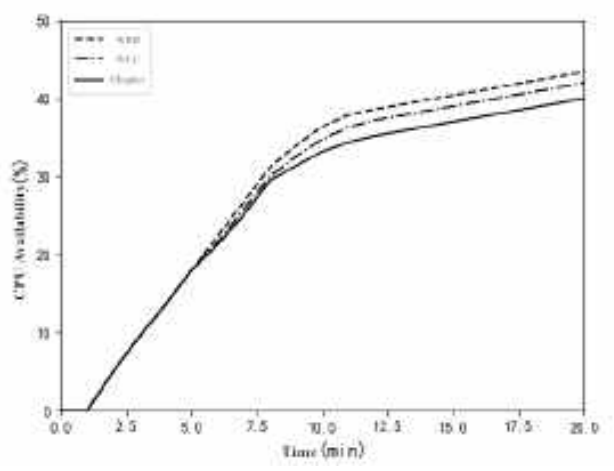

Figure 3.4 CPU utilization

\section{SUMMARY}

For the research of load balancing based on WEB cluster, this chapter proposes a WEB load balancing algorithm based on resource request division. It divides the request resources into static request resources and dynamic request resources, and caches the static request resources into memory to improve access efficiency. By analyzing the complexity of the algorithm and the effect of load balancing, and combining experiments to verify that the algorithm proposed in this chapter is superior to the WRR algorithm and LC algorithm in both average throughput and load balancer CPU utilization.

\section{REFERENCES}

[1]. Cardellini V, Colajanni M, Yu P S. Dynamic Load Balancing on Web-Server Systems[J].Internet computing IEEE, 2015, 3(3):28-39.

[2]. Liu Shengnan, Wang Shilin.Improvement of Dynamic Load Balancing Strategy for Web Services in VirtualEnvironment [J] .Computer Engineering and Science,2015,37(9):1607-1613

[3]. Menzel M, Ranjan R, Wang L, et al. CloudGenius: A Hybrid Decision Support Method for Automating the Migration of Web Application Clusters to Public Clouds[J]. IEEE Transactionson Computers, 2015, 64(5): 1336- 1348.

[4]. Cardellini V, Colajanni M, Yu P S. Dynamic Load Balancing on Web-Server Systems[J].Internet computing IEEE, 2015, 3(3):28-39.

[5]. Pu Xiaøyang.Research on Load Balancing of High Concurrency Web Services Based on Nginx and Redis [J]. Neijiang Technology,2016,(01):40+33.

[6]. Wu Yanqing.A load balancing mesh architecture based on Nginx, Memcached, and Tomcatt [P] .Shandong:CN104618508A,2015-05-13.

[7]. Wang Wenjun.Research and improvement of load balancing scheme based on Nginx server cluster [J]. electronic world,2017(02):179-181.

[8]. GUAN Chuan.Improvement and Implementation of Load Balancing Strategy for Web Server Based on Nginx [D] .Southwest Jiaotong University,2017. 